\title{
Dynamic Alterations of Rat Nucleus Accumbens Dendritic Spines over 2 Months of Abstinence from Extended-Access Cocaine Self-Administration
}

\author{
Daniel T Christian ${ }^{1,2}$, Xiaoting Wang',2, Eugenia L Chen', Lakshya K Sehgal', Michael N Ghassemlou', \\ Julia J Miao', Derenik Estepanian', Cameron H Araghi', Grace E Stutzmann' and Marina E Wolf*, \\ 'Department of Neuroscience, The Chicago Medical School at Rosalind Franklin University of Medicine and Science, North Chicago, IL, USA
}

\begin{abstract}
Chronic cocaine exposure influences the density and morphology of dendritic spines on medium spiny neurons (MSNs) in the nucleus accumbens (NAc), a critical brain region for cocaine craving. However, the relationship between spine plasticity and craving remains unclear. To study this relationship, we trained rats to self-administer cocaine using an extended-access regimen (6 h per day, 10 days); controls self-administered saline. Previously, a time-dependent intensification (incubation) of cue-induced cocaine craving has been demonstrated after withdrawal from this regimen; furthermore, $\mathrm{Ca}^{2+}$-permeable AMPA receptors (CP-AMPARs) increase in the NAc core after $\sim$ I month of withdrawal and thereafter mediate the expression of incubated craving. Although neither craving nor CP-AMPAR levels were measured in the present study, we killed rats at four withdrawal day (WD) time-points (WDI4, WD25, WD36, or WD60) selected to span the rising phase of incubation and the transition from low to high CP-AMPAR levels. MSNs were iontophoretically filled with Lucifer yellow and spines were analyzed with NeuronStudio software. Compared with saline controls, cocaine rats showed no changes in spine density or morphology in the NAc core on WDI4 or WD25. On WD36, approximately the withdrawal time when stable elevation of CP-AMPAR levels is detected, the cocaine group exhibited increased density of thin spines in the NAc core. By WD60, however, this effect had reversed: the density of thin spines was lower in cocaine rats compared with saline rats. In contrast, craving and CP-AMPAR levels remain high on WD60. We also assessed spine density on WD36 in the dorsolateral striatum, a region that is not implicated in incubation of cocaine craving and does not undergo CP-AMPAR plasticity. Here, the cocaine group exhibited a small leftward shift in the distribution of spine densities plotted as a cumulative distribution, opposite to the effect found in the NAc core. Overall, our results demonstrate changes in NAc core spines over 2 months of withdrawal but no simple relationship between the time dependency of these spine changes and the previously demonstrated time course of incubation of cocaine craving. However, they raise the possibility that CP-AMPAR accumulation in the NAc core occurs in a population of thin spines that emerges after $\sim 1$ month of withdrawal.

Neuropsychopharmacology (2017) 42, 748-756; doi:I0.1038/npp.2016.I68; published online 2 I September 2016
\end{abstract}

\section{INTRODUCTION}

Since the first demonstration that cocaine affects dendritic spines (Robinson and Kolb, 1999), there has been sustained interest in the role of structural plasticity in animal models of addiction, although no consensus has emerged regarding its functional consequence (Russo et al, 2010; Golden and Russo, 2012). The majority of studies on cocaine-induced structural plasticity have focused on the nucleus accumbens (NAc), which integrates excitatory inputs from cortical and limbic regions that influence motivated behaviors (Sesack and Grace, 2010), and have used non-contingent cocaine

\footnotetext{
*Correspondence: Dr ME Wolf, Department of Neuroscience, The Chicago Medical School at Rosalind Franklin University of Medicine and Science, 3333 Green Bay Road, North Chicago, IL 60064, USA, Tel: + I 847578 8659, Fax: + I 847578 8515, E-mail: marina.wolf@ rosalindfranklin.edu

${ }^{2}$ The first two authors contributed equally to this work.

Received 9 June 2016; revised 25 July 2016; accepted 16 August 2016; accepted article preview online 24 August 2016
}

regimens leading to behavioral sensitization. Many of these studies found increased spine density after non-contingent cocaine exposure (Golden and Russo, 2012). Recent evidence suggests that this is related to sensitization of cocaine's incentive-motivational rather than locomotor-activating effects (Wang et al, 2013).

Surprisingly, we are aware of only six studies of NAc dendritic spines after cocaine self-administration (see Discussion). Such studies are important because selfadministration models are required to investigate the relationship between spine plasticity and cocaine craving, and because excitatory synaptic transmission-which is expected to be related to spine plasticity-undergoes markedly different plasticity after withdrawal from noncontingent vs contingent cocaine (Wolf and Ferrario, 2010; Wolf, 2016). The aim of the present study was to analyze spine density and morphology in NAc medium spiny neurons (MSNs) after withdrawal from an extended-access cocaine self-administration regimen shown in many previous studies to elicit progressive intensification (incubation) 
of cue-induced cocaine craving after withdrawal/abstinence (these terms will be used interchangeably) (Pickens et al, 2011). Incubation of drug craving may help explain the persistence of cocaine craving even after prolonged abstinence (Pickens et al, 2011; Wolf, 2016) and has been demonstrated in human cocaine users (Parvaz et al, 2015).

Incubation of cocaine craving in rodents involves a time-dependent increase in $\mathrm{Ca}^{2+}$-permeable AMPA receptors (CP-AMPARs) in excitatory synapses on NAc core MSNs (Wolf, 2016). Normally present at low levels in NAc synapses, CP-AMPARs increase after $\sim 1$ month of withdrawal, strengthening excitatory transmission onto MSNs, and thereafter their activation is required for expression of incubated cocaine craving (Conrad et al, 2008; Purgianto et al, 2013; Loweth et al, 2014). The present study asked whether time-dependent structural plasticity occurs in NAc core that could support this strengthening of excitatory synaptic transmission. Accordingly, in contrast to previous studies that examined a single withdrawal time, we analyzed four withdrawal time-points, selected to span the incubation of cocaine craving (Lu et al, 2004) and the increase in NAc core CP-AMPAR levels (Wolf and Tseng, 2012). We also examined the NAc shell, which contributes to incubation (Wolf, 2016). As a control, we assessed the dorsolateral striatum (DLS), which contributes to cocaine seeking during abstinence but not its incubation (Pacchioni et al, 2011).

\section{MATERIALS AND METHODS}

\section{Drug Self-Administration}

All procedures were approved by our Institutional Animal Care and Use Committee. Adult male Sprague-Dawley rats (Harlan, Indianapolis, IN), weighing 250-275 g upon arrival, were housed 3 per cage under a reverse 12-h light-dark cycle with food and water freely available. As described previously (Conrad et al, 2008; Loweth et al, 2014), after at least 5 days of recovery from jugular catheter implantation, rats were trained (6 h per day, 10 days) in operant chambers equipped with two nose-poke holes (Med Associates, St Albans, VT). Active hole responding delivered an infusion of saline or cocaine $(0.5 \mathrm{mg} / \mathrm{kg}$ per $100 \mu \mathrm{l}$ over $3 \mathrm{~s})$ paired with a $30-\mathrm{s}$ light cue inside the nose-poke hole. Inactive hole responding had no consequences. Different groups of saline and cocaine rats were killed on withdrawal day 14 (WD14) (saline, $n=5$; cocaine, $n=4$ ), WD25 (saline, $n=5$; cocaine, $n=5$ ), WD36 (saline, $n=5$; cocaine, $n=4$ ), or WD60 (saline, $n=5$; cocaine, $n=5$ ).

\section{Brain Fixation}

Rats were transcardially perfused with cold $1 \%$ paraformaldehyde in phosphate-buffered saline (PBS), $\mathrm{pH} 7.4$, for $1 \mathrm{~min}$, followed by cold $4 \%$ paraformaldehyde with $0.125 \%$ glutaraldehyde in PBS for $12 \mathrm{~min}$. Brains were removed and postfixed in the same fixative for $12 \mathrm{~h}$. Timing was determined from preliminary experiments comparing postfixation durations from 6 to $24 \mathrm{~h}$ to optimize Lucifer yellow visualization and cellular retention. Fixed brains were sectioned into $200 \mu \mathrm{m}$ thick slices.

\section{Microinjection of Lucifer Yellow}

A 5\% (100 mM) Lucifer yellow solution (Invitrogen, Carlsbad, CA) was injected into MSNs using an iontophoretic cell loading procedure (1-5 nA for 1-3 min; Meredith et al, 1992; Dumitriu et al, 2011) (see Supplementary Materials). Sections were then mounted on slides with a $120 \mu \mathrm{m}$ spacer and coverslipped before imaging.

\section{Confocal Imaging and Spine Analysis}

To ensure that we analyzed distal dendrites, which show robust cocaine-induced plasticity (Robinson and Kolb, 2004; Ferrario et al, 2005), we imaged dendritic segments located $>50 \mu \mathrm{m}$ from the soma. We selected segments that did not overlap with other segments. As described previously (Dumitriu et al, 2011, 2012) and detailed in Supplementary Materials, images were acquired on a confocal LSM 510 (Carl Zeiss) and analyzed with NeuronStudio software, which automatically classifies spines as thin, mushroom, or stubby based on head-to-neck diameter ratio, length-to-head diameter ratio, and head diameter.

\section{Statistical Analysis}

Spine densities were calculated as the number of total spines or morphologically defined spines (thin, stubby, mushroom) divided by the length of the dendritic segment. Cumulative distributions encompass all spines from every dendritic segment for all animals in the experimental group. Distribution differences were evaluated using the KolmogorovSmirnov (K-S) test. For bar graphs, spine densities from all dendritic segments were averaged for each animal, and these values were averaged to determine the mean \pm S.E.M. for all animals in the group. Comparisons of total spine density between cocaine and saline groups at a particular withdrawal time were performed using unpaired Student's $t$-tests. Comparisons of multiple spine subtypes were performed using two-way ANOVAs with Bonferroni post hoc tests. Significance was set at $p<0.05$ (NS, not significant).

\section{RESULTS}

Rats self-administered saline or cocaine using an extendedaccess regimen (Figure 1) that is well established as leading to incubation of cocaine craving ( $\mathrm{Lu}$ et al., 2004; Conrad et al., 2008; Purgianto et al., 2013; Loweth et al., 2014). For reasons discussed previously, this regimen does not always lead to escalation of intake (see Purgianto et al., 2013; Wolf, 2016), consistent with evidence that escalation and incubation involve distinct changes in NAc MSN firing (Guillem et al., 2014). Rats were perfused for spine analysis on WD14 (well before CP-AMPAR accumulation), WD25 (just before onset of CP-AMPAR accumulation), WD36 (shortly after maximal CP-AMPAR levels are attained), or WD60 (stably elevated CP-AMPAR expression) (Wolf and Tseng, 2012). Behaviorally, cue-induced cocaine craving, measured after a nearly identical regimen, progressively intensifies (incubates) from WD1 through $\sim$ WD30, reaching a plateau that persists at least through WD90 (Lu et al, 2004). 


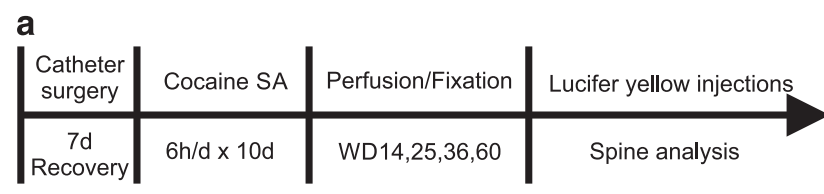

b

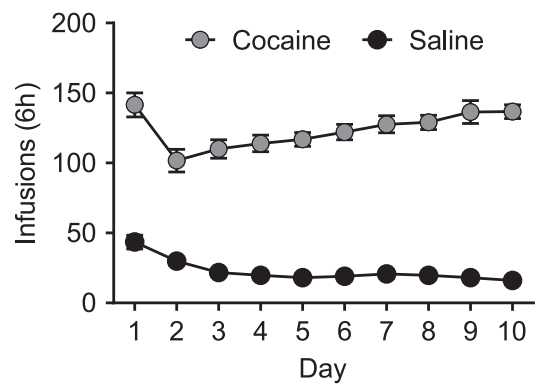

Figure I Experimental design and self-administration training data. (a) Timeline. (b) Number of infusions during self-administration sessions (mean \pm S.E.M.; saline, $n=20$ rats; cocaine, $n=18$ rats). (c) Representative I0x single plane confocal images showing nucleus accumbens core (AcbC) and shell (AcbSh) (top) and dorsolateral striatum (DLS) (bottom), labeled according to Paxinos and Watson (2007), following iontophoretic injection of Lucifer yellow into medium spiny neurons (MSNs). aca, Anterior commissure; ec, external capsule; SA, self-administration.

\section{Total Spine Density in the NAc Core}

For each experimental group, total spine density data were analyzed in two ways: (1) the average total spine density was calculated per animal and then averaged for each group, and (2) the distribution of total spine densities was plotted as a cumulative frequency (this included all spines on each analyzed dendrite from all animals in each group). Our measures of total spine density in saline controls correspond well to those reported previously (eg, Dumitriu et al, 2012). We began by comparing spine density in the NAc core of each cocaine group to the average number of cocaine infusions during training and found no significant correlations (Supplementary Table 1). The remainder of our analysis focused on potential relationships between spine density and withdrawal time. In saline rats, ANOVA revealed no change in spine density over the four withdrawal times, as expected $(\mathrm{F}(3,16)=2.30, p=0.12)$. However, cocaine rats showed a significant change over withdrawal $(\mathrm{F}(3,14)=6.05, p<0.01)$, with Bonferroni tests indicating significantly increased spine density on WD36 compared with both WD25 $(t(14)=3.10, p<0.05)$ and WD60 $(t(14)=3.91, p<0.01)$.

Based on these findings, we compared saline and cocaine groups on each WD. On WD14, these groups showed no difference in total spine density (Student's $t$-test, $t(7)=0.42$, $p=0.68)$ or the cumulative frequency distribution of spine densities for all dendritic segments $(\mathrm{K}-\mathrm{S}$ test $=0.11, p=0.59)$ (Figure 2a). Similarly, cocaine and saline groups killed on WD25 did not differ in total spine density $(t(8)=0.57$, $p=0.57$ ) or cumulative distribution (K-S test $=0.07$, $p=0.87$ ) (Figure $2 b)$. However, on WD36, total spine density was significantly increased in the cocaine group $(t(7)=3.52$, $p<0.01)$. This occurred in both low- and high-density segments as indicated by a generalized rightward shift in the cumulative distribution $(\mathrm{K}-\mathrm{S}$ test $=0.26, \quad p<0.0001)$ (Figure 2c). By WD60, the increase in total spine density observed on WD36 had reversed and in fact there was a trend towards a small decrease in cocaine rats $(t(8)=1.86$, $p=0.09)$. Substantiating this trend, the cumulative distribution was leftward shifted (K-S test $=0.27$, $p<0.0005)$, although not for those segments with the highest spine density (Figure 2d). These results suggest that an increase in spines occurs in the NAc core between WD25 and WD36 and a loss of spines occurs between WD36 and WD60.

\section{Spine Morphology in the NAc Core}

Next, we determined if cocaine withdrawal differentially affected thin, mushroom, and stubby spines. All dendritic segments for each animal were averaged; then, data from all animals in each group were averaged to provide a density measure for each group. On each of the four WDs, two-way ANOVA (treatment $\times$ spine type) revealed a significant main effect for spine type (WD14: $\mathrm{F}(2,21)=353.9$, $p<0.0001 ; \quad W D 25: \quad \mathrm{F}(2,24)=449.7, \quad p<0.0001 ; \quad$ WD36: $\mathrm{F}(2,21)=468.0, \quad p<0.0001 ; \quad$ WD60: $\quad \mathrm{F}(2,24)=348.30$, $p<0.0001)$. This simply reflects the fact that thin spines were more prevalent than other spine types in all experimental groups.

On WD14, we found no significant main effect of drug treatment $(\mathrm{F}(1,21)=0.26, p=0.61)$ and no significant treatment $\times$ spine-type interaction $(\mathrm{F}(2,21)=0.34, p=0.71)$. Confirming these findings, post hoc analysis using Bonferroni tests revealed no group differences in spine density for thin $(t(21)=0.24$, NS), stubby $(t(21)=0.90$, NS), or mushroom $(t(21)=0.25$, NS) spines (Figure 3a). Similarly, on WD25, we found no main effect of drug treatment $(\mathrm{F}(1,24)=0.16, p=0.69)$ and no interaction $(\mathrm{F}(1,24)=0.16$, $p=0.69$ ). Post hoc Bonferroni tests found no group difference in density of thin $(t(24)=0.78$, NS), stubby $(t(24)=0.14, \mathrm{NS})$, or mushroom spines $(t(24)=0.04$, NS) (Figure $3 b$ ).

On WD36, however, we found a significant main effect of drug treatment $(\mathrm{F}(1,21)=13.66, p=0.001)$, as well as a significant treatment $\times$ spine-type interaction $(\mathrm{F}(2,21)=7.43$, $p<0.005)$. The main driver of these effects was an increase in the density of thin spines in the cocaine group (Bonferroni, $t(21)=5.21, p<0.05)$; no group differences were found for stubby $(t(21)=0.04, \mathrm{NS})$ or mushroom $(t(21)=1.13$, NS) spines (Figure 3c).

On WD60, there was a small main effect of drug treatment $(\mathrm{F}(1,24)=4.39, p=0.04)$, consistent with results in Figure 2d, but no significant interaction $(\mathrm{F}(2,24)=1.73, p=0.19)$. However, post hoc analysis revealed a significant decrease in the density of thin spines in the cocaine animals $(t(24)=2.70, \quad p<0.05)$, but no differences for stubby $(t(24)=0.73, \mathrm{NS})$ or mushroom $(t(24)=0.19$, NS) spines (Figure 3d).

Overall, these findings indicate that thin spines account for the increase in total spine density occurring between WD25 and WD36 in the NAc core and the decrease in total spine density occurring between WD36 and WD60. 
a
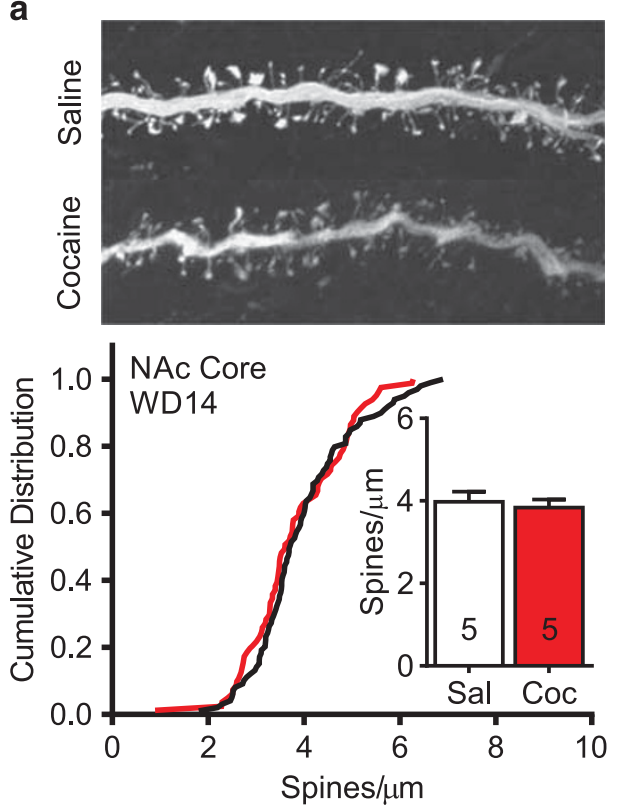

C
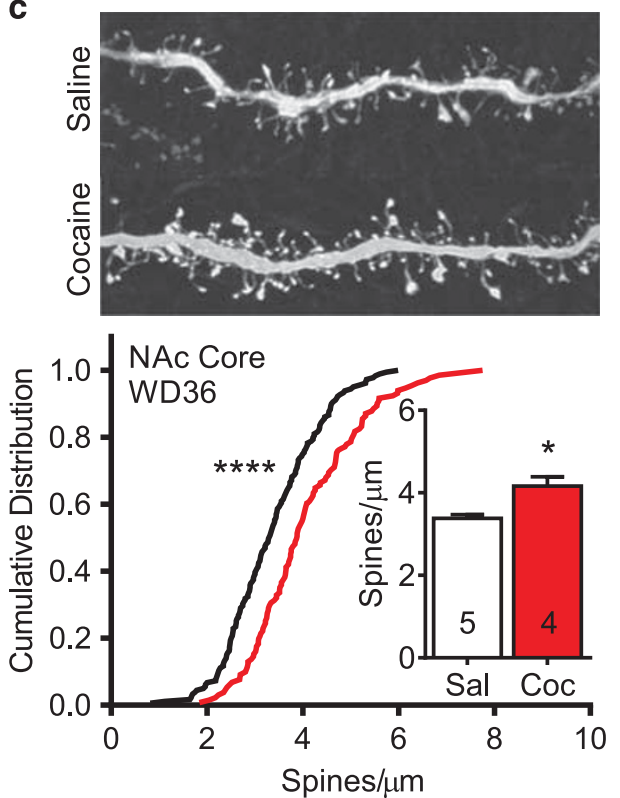
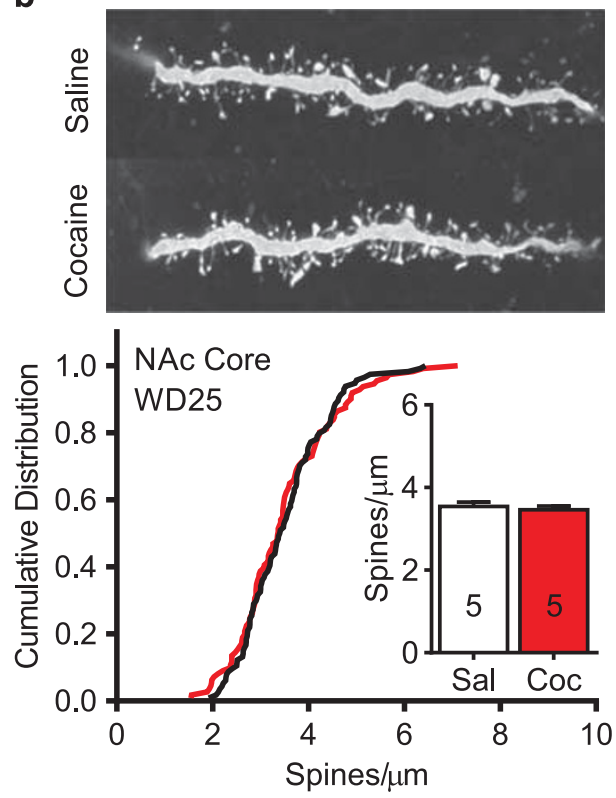

d
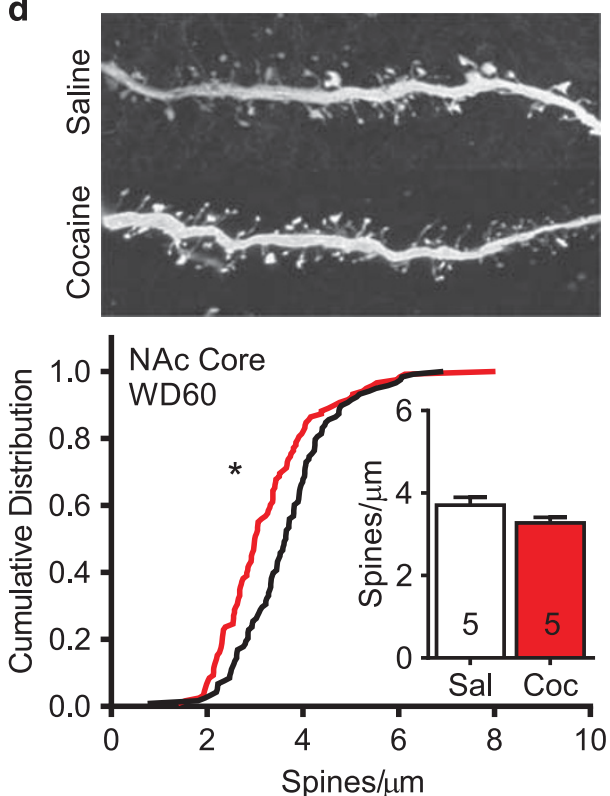

Figure 2 Total spine density in medium spiny neurons of the nucleus accumbens (NAc) core undergoes time-dependent alterations after withdrawal from cocaine self-administration. (a-d) Top images show representative projected z-stack images (I00x) from each experimental group. Bottom graphs show cumulative frequency distributions for every dendritic segment from all animals in a group (black line $=$ saline; red line $=$ cocaine). Inset graphs present mean total spine density averaged first by animal and then for the group. Numbers in bars represent the number of animals in each condition. (a) On withdrawal day 14 (WD I4), no differences in total spine density or their cumulative distribution were observed between saline and cocaine groups. (b) On WD25, no differences in total spine density or their cumulative distribution were observed between saline and cocaine groups. (c) On WD36, a significant increase in total spine density and a significant shift of the cumulative distribution to the right were observed in the cocaine group. (d) On WD60, a reversal occurred such that the cumulative distribution of spines in the cocaine group was shifted significantly to the left. $* p<0.05$ and $* * * * * 00.000$ l.

\section{Spine Density and Morphology in the NAc Shell}

Using tissue from the same animals used to analyze the NAc core, we examined dendritic spines at each withdrawal time in the NAc shell. Overall, our results indicate no significant effect of cocaine withdrawal (Supplementary Figures 1 and 2), although interpretation of these data is complicated by a small difference in spine density between the saline WD14 group and other saline groups, as discussed in Supplementary Material and the Discussion section.

\section{Spine Analysis in DLS on WD36}

The DLS was selected as a control region because it is not implicated in incubation of cocaine craving or related CP-AMPAR plasticity (see Discussion). We injected Lucifer yellow into DLS neurons from the rats killed on WD36, the time-point at which spine density was significantly increased in the NAc core. Total spine density in DLS did not differ significantly between cocaine and saline groups $(t(7)=1.25$, $p=0.25)$. However, there was a small but significant leftward 

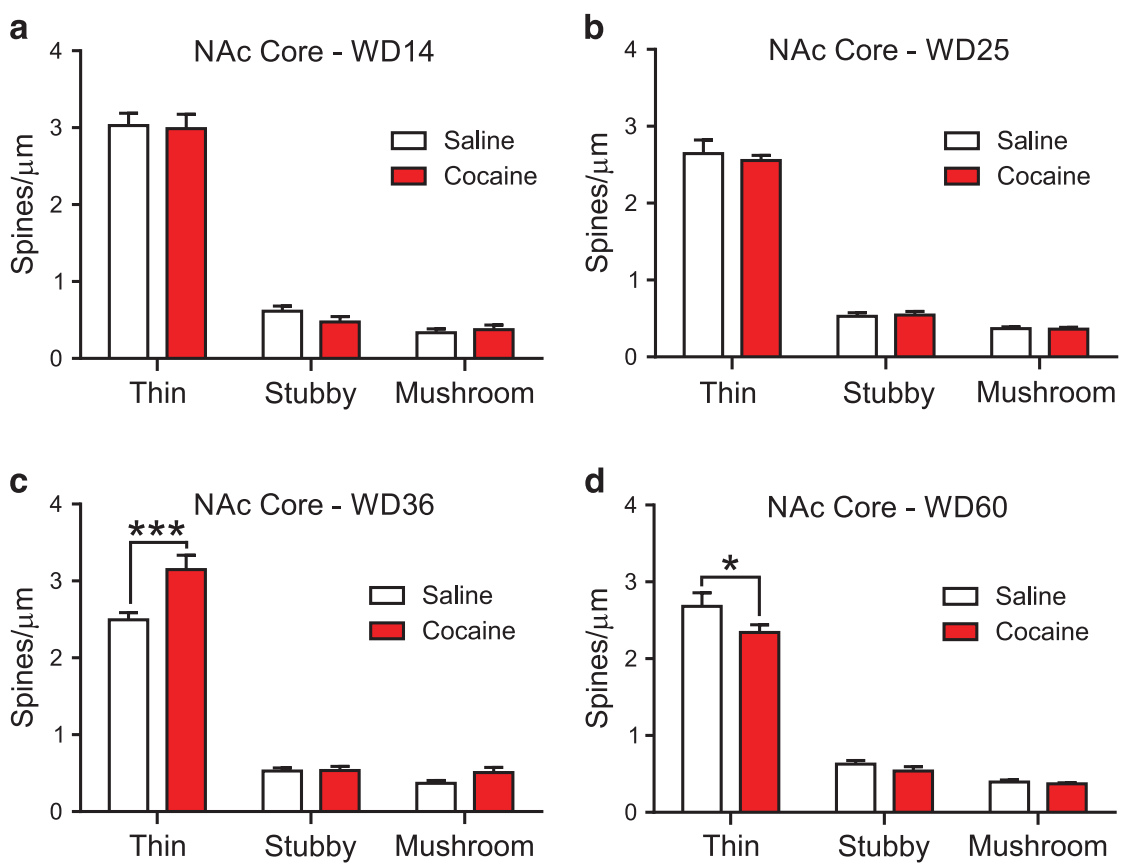

Figure 3 Cocaine self-administration regulates the number of thin spines in the nucleus accumbens (NAc) core in a manner that depends on the length of withdrawal. (a) On withdrawal day I4 (WDI4), the density of dendritic spine subtypes did not differ between saline and cocaine groups. (b) On WD25, the density of dendritic spine subtypes did not differ between saline and cocaine groups. (c) On WD36, the cocaine group exhibited a significant increase in the density of thin spines in the NAc core in comparison with saline controls. This increase in thin spine density is likely the primary driver behind the increase in total spine density on WD36 (Figure 2c). (d) On WD60, there was a significant reduction in the density of thin spines in cocaine animals compared with saline controls, but no change in the density of any other dendritic spine subtype. Thus, alterations in thin spines appear to underlie results presented in Figure $2 \mathrm{~d}$. * $p<0.05$ and $* * * * p<0.001$.

shift in the cumulative distribution of spine density in cocaine rats $(\mathrm{K}-\mathrm{S}$ test $=0.19, \quad p<0.01) \quad$ (Figure 4a). Analysis of spine subtypes in saline and cocaine rats revealed a significant main effect of spine type (two-way ANOVA, $\mathrm{F}(2,21)=124.00, \quad p<0.0001)$, again reflecting greater prevalence of thin spines in both experimental groups, but no significant effect of drug treatment $(\mathrm{F}(1,21)=2.61$, $p=0.12)$ and no significant interaction $(\mathrm{F}(2,21)=0.65$, $p=0.53$ ). Post hoc analysis found no differences between cocaine and saline animals in the density of any spine subtype: thin $(t(21)=1.69, \mathrm{NS})$, stubby $(t(21)=1.01$, NS), or mushroom $(t(21)=0.08$, NS) (Figure $4 \mathrm{~b})$. Overall, these findings reveal a small decrease in spine density in the DLS on WD36.

\section{DISCUSSION}

Our main aim was to analyze spine density and morphology in the NAc core at withdrawal times (WD14, WD25, WD36, and WD60) from extended-access cocaine selfadministration that span the time-dependent increase in cue-induced cocaine craving (incubation) (Lu et al, 2004) and the transition from low to 'incubated' levels of CP-AMPARs (Wolf and Tseng, 2012).

\section{Spine Plasticity in the NAc Core and Dorsal Striatum During Incubation}

After the same regimen used herein, CP-AMPAR levels in the NAc core increase between WD25 and WD35, and then remain stably elevated (Wolf and Tseng, 2012). Although other mechanisms must account for incubation during the first month of withdrawal, once CP-AMPARs increase in the NAc core, their activation is required for expression of incubation (Conrad et al, 2008; Loweth et al, 2014), consistent with other evidence that enhanced activation of NAc core MSNs is critical for incubation (Hollander and Carelli, 2005, 2007; Guillem et al, 2014). Here we observed no differences in NAc core spine density or morphology between cocaine and saline rats on WD14 or WD25. On WD36, just after CP-AMPARs are maximally elevated, cocaine rats showed increased spine density, largely attributable to thin spines. However, whereas CP-AMPAR levels remain high through at least WD90 (Wolf and Tseng, 2012), the cumulative frequency distribution revealed that spine density in cocaine rats fell slightly below control levels on WD60.

These results suggest that structural plasticity may coincide with CP-AMPAR elevation in the NAc core after withdrawal. It is possible that the new thin spines observed on WD36 are the site of CP-AMPAR incorporation and then persist, whereas other spines are lost (accounting for reversal of the increase in spine density by WD60). Thin spines can be immature and plastic, but they can also form persistent synapses (Holtmaat and Svoboda, 2009). Of course, there are alternative possibilities. For example, CP-AMPARs may be added, around WD30, to a population of pre-existing spines that remains relatively stable throughout subsequent withdrawal, with the gain in spines by WD36 and the loss by WD60 involving spines different from those that undergo CP-AMPAR plasticity. 
a
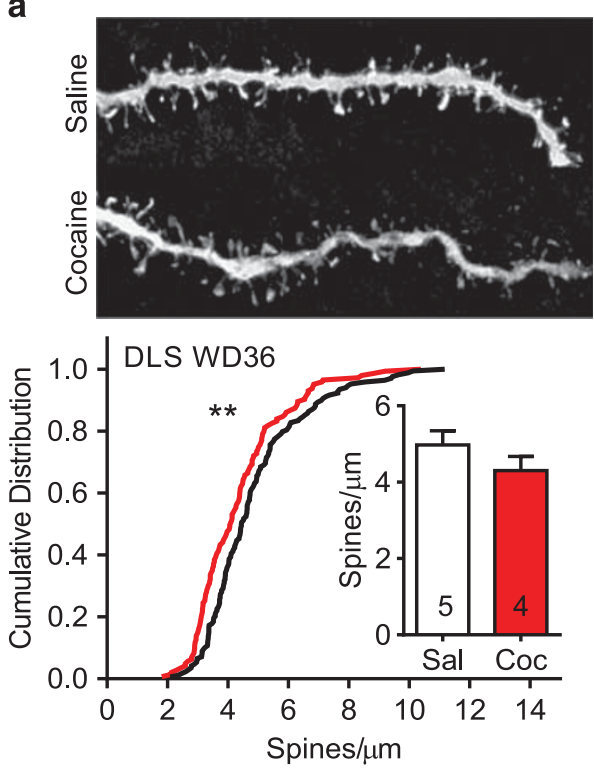

b

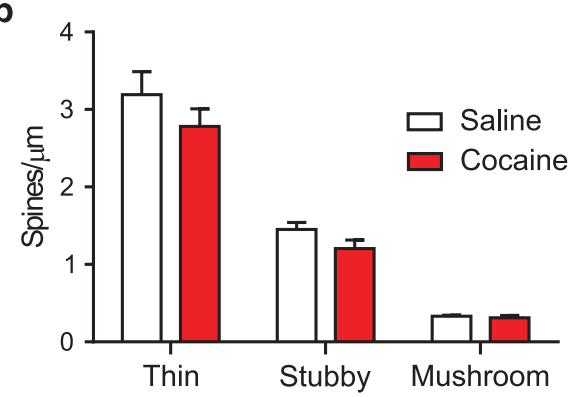

Figure 4 Spine density in the dorsolateral striatum (DLS) on withdrawal day 36 (WD36) following saline or cocaine self-administration. (a) Top images show representative projected z-stack images (I00x) from each experimental group. Bottom graphs show cumulative distributions for every dendritic segment from all animals in a group (black line=saline; red line $=$ cocaine). Inset graphs present mean total spine density averaged first by animal and then for the group. Numbers in bars represent the number of animals in each condition. On WD36, total spine density did not differ between saline and cocaine groups. However, the cumulative distribution for cocaine segments was significantly shifted to the left. (b) No group difference in the density of any dendritic spine subtype was found in the DLS on WD36. ${ }^{* *} p<0.01$.

The DLS has been linked to cocaine seeking during forced abstinence but not its incubation (Fuchs et al, 2006; Pacchioni et al, 2011). Consistent with this, the present cocaine regimen does not elevate CP-AMPAR levels in the DLS (DT Christian, ME Wolf, KY Tseng, unpublished data). Therefore, spine changes that occur in NAc, but not DLS, are candidates for mediating plasticity related to incubation and CP-AMPAR accumulation. We found a decrease in thin spines in the DLS on WD36, that is, an opposite effect to that observed in the NAc core of the same animals. These results are consistent with the possibility that NAc core spine changes are related to CP-AMPAR plasticity.

\section{Spine Changes in the NAc Shell During Incubation}

In the NAc shell, no difference between cocaine and saline groups was found on WD25, WD36, or WD60; however, spine density was relatively lower in cocaine rats on WD14. This is difficult to interpret because it appears to be driven by higher spine density in the saline group on WD14 compared with other saline withdrawal times (see Supplementary Material). CP-AMPARs accumulate in the shell during forced abstinence from cocaine self-administration regimens (Mameli et al, 2009; Pascoli et al, 2014; Terrier et al, 2015; Neumann et al, 2016), including the regimen used herein (McCutcheon et al, 2011), and this contributes to incubated cocaine seeking (Lee et al, 2013; Ma et al, 2014). However, compared with the core, less is known about the time course of CP-AMPAR elevation in the shell, although it may begin as early as WD10 (Lee et al, 2013). Furthermore, whereas CP-AMPARs upregulate in all core MSNs, this occurs in only about half of shell MSNs (McCutcheon et al, 2011), apparently reflecting both pathway-specific and D1 receptor (D1R)/D2 receptor (D2R) MSN differences (Lee et al, 2013; Ma et al, 2014; Pascoli et al, 2014; Terrier et al, 2015). This heterogeneity increases the difficulty of detecting spine plasticity related to CP-AMPAR plasticity.

Although we failed to detect cocaine-induced increases in spine density in the NAc shell, two studies have found such increases after 1 month of abstinence from cocaine self-administration (Robinson et al, 2001; Ferrario et al, 2005), as described in more detail in the next section. We speculate that the difference may be due to the longer duration of their regimens (the number of sessions ranged from 21 to 37) compared with our regimen (10 sessions).

\section{Comparison with Other Cocaine Studies}

Most studies of NAc dendritic spines after cocaine exposure have used non-contingent cocaine regimens (see Introduction). Although results vary depending on multiple variables, many studies have found increases in spine density that are detectable soon after discontinuing cocaine administration and then persist for weeks (Golden and Russo, 2012). In contrast, there have been only six studies of NAc spines after cocaine self-administration, and only three of these examined the effect of withdrawal. Robinson et al. (2001) found a small but significant increase in the NAc shell spine density after 1 month of withdrawal from cocaine self-administration ( 1 or $2 \mathrm{~h}$ per day $\times 30$ days), but the core was not examined. Ferrario et al. (2005) compared rats after 1 month of withdrawal from extended-access ( $6 \mathrm{~h}$ per day) vs limited-access ( $1 \mathrm{~h}$ per day) cocaine self-administration. After limited access, spine density increased to a similar extent in the core and shell; after extended access, spine changes were more pronounced in the core and were correlated with greater locomotor sensitization and cocaine seeking. Bock et al. (2013) did not examine spine density, but they found an increase in spine head width on NAc core D1R MSNs after cocaine self-administration, which was associated with synaptic potentiation; a subset of rats exhibiting lower vulnerability to compulsive cocaine use also showed synaptic potentiation onto D2R MSNs, but spines were not assessed (cocaine self-administration: $2 \mathrm{~h}$ per day for an average of 30 days; withdrawal times ranged from 1 to 38 days).

Overall, the only directly comparable results (Ferrario et al, 2005) are consistent with our finding of increased spine density in the NAc core after $\sim 1$ month of withdrawal. 
We emphasize that no prior studies have evaluated NAc spine density at either shorter or longer withdrawals from cocaine self-administration. Although it is generally assumed that cocaine exposure leads to a relatively rapid increase in spine density in the NAc core, the evidence for this idea comes exclusively from studies of non-contingent cocaine exposure (Golden and Russo, 2012). Of course, we cannot rule out the possibility that our cocaine regimen led to very rapid changes in spine density that dissipated by WD14; this should be examined in future studies. However, our aim here was to test for spine plasticity accompanying progressive changes in craving and AMPAR transmission in later withdrawal phases.

Three recent studies reported rapid, transient increases in spine density in the NAc core during reinstatement of cocaine seeking following extinction training (Gipson et al, 2013; Shen et al, 2014; Stankeviciute et al, 2014). It is possible that the same occurs during a cue-induced seeking test in 'incubated rats', although this cannot be assumed, as extinction training causes its own plasticity (eg, Self et al, 2004) that could influence the spine response during reinstatement. We did not examine spines after a seeking test in the present study because we were interested in spine plasticity that might correlate with CP-AMPAR accumulation, and the latter does not require a seeking test to become evident.

\section{AMPAR Subunit Composition and Spine Morphology}

In many instances of synaptic plasticity, there is a correlation between the magnitude of the excitatory synaptic response and spine expansion (Sala and Segal, 2014). This may seem at odds with our findings in the incubation model, where electrophysiological studies reveal persistently strengthened synapses (Conrad et al, 2008), but this does not coincide with a shift towards larger diameter (mushroom) spines (present results). Of course, this could be explained if CP-AMPARs are added to new spines. However, if CP-AMPARs are instead added to existing spines, the observed lack of spine expansion could reflect the fact that synapses are strengthened through incorporation of CP-AMPARs rather than $\mathrm{Ca}^{2+}$-impermeable AMPARs (CI-AMPARs). The higher conductance of CP-AMPARs may offer a way to enhance synaptic strength without the need for an overall increase in the total number of AMPARs in the postsynaptic density, obviating the need for increased spine volume (Beique et al, 2011; Soares et al, 2013).

\section{Silent Synapses and Spine Plasticity}

In the NAc shell, an increase in silent synapses (ie, synapses containing NMDARs but no AMPARs) has been demonstrated on WD1 from a regimen leading to incubation of cocaine craving; these silent synapses are unsilenced, via incorporation of either CP-AMPARs or CI-AMPARs depending on the pathway, by WD45 (Lee et al, 2013; Ma et al, 2014; Neumann et al, 2016). Also depending on the pathway, this silent synapse-based remodeling can either promote or oppose incubation of cocaine craving (Lee et al, 2013; Ma et al, 2014). Silent synapses can form through addition of NMDARs to new synaptic sites or loss of AMPARs from existing synapses, and the relationships of these events to spine density/morphology are not well defined (Kerchner and Nicoll, 2008). Our earliest timepoint (WD14) was probably too late to detect spine plasticity related to cocaine-induced silent synapse formation, and our results at later withdrawal times are difficult to relate to unsilencing because all that is known about the time course of unsilencing is that it may begin by WD10 in some pathways (Lee et $a l, 2013$ ) and is complete by WD45 (see above).

\section{Caveats Imposed by Heterogeneity of MSN}

D1R/substance P- and D2R/enkephalin-expressing MSNs can exhibit different synaptic plasticity following cocaine self-administration (eg, Bock et al, 2013) and forced abstinence from cocaine self-administration (Pascoli et al, 2014; Terrier et al, 2015). Non-contingent cocaine administration can increase spine density in both populations, but some studies suggest that this may be more long-lived in D1R-MSNs (eg, Lee et al, 2006; see Golden and Russo (2012) for review). We are attempting to develop immunohistochemical methods for post hoc identification of Lucifer yellow-filled cells as D1R/substance P- or D2R/ enkephalin-positive, but it has been very challenging to develop a method compatible with Lucifer yellow filling. However, if spine density changes are associated with CP-AMPAR accumulation, we predict similar changes in D1R-MSNs and D2R-MSNs in the NAc core, as CP-AMPAR accumulation is detected in nearly all core MSNs recorded after $>$ WD35 (McCutcheon et al, 2011). The shell is more heterogeneous (see above). In addition to $\mathrm{D} 1 \mathrm{R} / \mathrm{D} 2 \mathrm{R}$ distinctions, it is important to recall that MSNs are heterogeneous in their processing of rewardrelated information (eg, Wheeler and Carelli, 2009) and that cocaine seeking is regulated by small ensembles of MSNs (eg, Cruz et al, 2014) and specific pathways (eg, Lee et al, 2013; Ma et al, 2014; Pascoli et al, 2014). All of these factors may contribute to difficulties in establishing links between spine and behavioral changes after cocaine exposure.

\section{CONCLUSIONS}

First, there is no clear relationship between NAc spine density (present study) and the level of cue-induced cocaine craving (prior studies) over 2 months of withdrawal from extended-access cocaine self-administration. Second, based on the previously established time course of CPAMPAR accumulation, our results suggest that a transient increase in spine density may accompany the shift from low to higher CP-AMPAR levels in NAc core synapses that occurs after $\sim 1$ month of withdrawal. However, spine density subsequently normalizes, indicating that elevated CP-AMPARs (and incubated craving) are subsequently maintained in the absence of a sustained increase in spine density. Functional studies at the single spine level will be required to unravel the relationship between morphological and glutamate receptor plasticity during incubation of craving. 


\section{FUNDING AND DISCLOSURE}

This research was supported by DA009621, DA015835, and DA029099 (to MEW); DA034943 (to GES); and postdoctoral NRSA DA036963 (to DTC). The authors declare no conflict of interest.

\section{ACKNOWLEDGMENTS}

We are very grateful to Drs Scott Russo and Sam Golden for teaching us methods for dendritic spine analysis, and to Dr Gloria Meredith for assistance in setting up the technique and for use of her equipment for iontophoretic injections. We also acknowledge the Confocal Facility at Rosalind Franklin University of Medicine and Science for technical assistance.

\section{REFERENCES}

Beique JC, Na Y, Kuhl D, Worley PF, Huganir RL (2011). Arc-dependent synapse-specific homeostatic plasticity. Proc Natl Acad Sci U S A 108: 816-821.

Bock R, Shin JH, Kaplan AR, Dobi A, Markey E, Kramer PF et al (2013). Strengthening the accumbal indirect pathway promotes resilience to compulsive cocaine use. Nat Neurosci 16: 632-638.

Conrad KL, Tseng KY, Uejima JL, Reimers JM, Heng LJ, Shaham Y et al (2008). Formation of accumbens GluR2-lacking AMPA receptors mediates incubation of cocaine craving. Nature 454: $118-121$.

Cruz FC, Babin KR, Leao RM, Goldart EM, Bossert JM, Shaham Y et al (2014). Role of nucleus accumbens shell neuronal ensembles in context-induced reinstatement of cocaine-seeking. J Neurosci 34: 7437-7446.

Dumitriu D, Laplant Q, Grossman YS, Dias C, Janssen WG, Russo SJ et al (2012). Subregional, dendritic compartment, and spine subtype specificity in cocaine regulation of dendritic spines in the nucleus accumbens. J Neurosci 32: 6957-6966.

Dumitriu D, Rodriguez A, Morrison JH (2011). High-throughput, detailed, cell-specific neuroanatomy of dendritic spines using microinjection and confocal microscopy. Nat Protocols 6: 1391-1411.

Ferrario CR, Gorny G, Crombag HS, Li Y, Kolb B, Robinson TE (2005). Neural and behavioral plasticity associated with the transition from controlled to escalated cocaine use. Biol Psychiatry 58: 751-759.

Fuchs RA, Branham RK, See RE et al (2006). Different neural substrates mediate cocaine seeking after abstinence versus extinction training: a critical role for the dorsolateral caudateputamen. J Neurosci 26: 3584-3588.

Gipson CD, Kupchik YM, Shen H, Reissner KJ, Thomas CA, Kalivas PW (2013). Relapse induced by cues predicting cocaine depends on rapid, transient synaptic potentiation. Neuron 77: $867-872$

Golden SA, Russo SJ (2012). Mechanisms of psychostimulantinduced structural plasticity. Cold Spring Harb Perspect Med 2: 10.

Guillem K, Ahmed SH, Peoples LL (2014). Escalation of cocaine intake and incubation of cocaine seeking are correlated with dissociable neuronal processes in different accumbens subregions. Biol Psychiatry 76: 31-39.

Hollander JA, Carelli RM (2005). Abstinence from cocaine selfadministration heightens neural encoding of goal-directed behaviors in the accumbens. Neuropsychopharmacology 30: 1464-1474.

Hollander JA, Carelli RM (2007). Cocaine-associated stimuli increase cocaine seeking and activate accumbens core neurons after abstinence. J Neurosci 27: 3535-3539.
Holtmaat A, Svoboda K (2009). Experience-dependent structural synaptic plasticity in the mammalian brain. Nat Rev Neurosci 10: 647-658.

Kerchner GA, Nicoll RA (2008). Silent synapses and the emergence of a postsynaptic mechanism for LTP. Nat Rev Neurosci 9: $813-825$.

Lee BR, Ma YY, Huang YH, Wang X, Otaka M, Ishikawa M et al (2013). Maturation of silent synapses in amygdala-accumbens projection contributes to incubation of cocaine craving. Nat Neurosci 16: 1644-1651.

Lee KW, Kim Y, Kim AM, Helmin K, Nairn AC, Greengard P (2006). Cocaine-induced dendritic spine formation in D1 and D2 dopamine receptor-containing medium spiny neurons in nucleus accumbens. Proc Natl Acad Sci USA 103: 3399-3404.

Loweth JA, Scheyer AF, Milovanovic M, LaCrosse AL, FloresBarrera E, Werner CT et al (2014). Synaptic depression via mGluR1 positive allosteric modulation suppresses cue-induced cocaine craving. Nat Neurosci 17: 73-80.

Lu L, Grimm JW, Hope BT, Shaham Y (2004). Incubation of cocaine craving after withdrawal: a review of preclinical data. Neuropharmacology 47(Suppl 1): 214-226.

Ma YY, Lee BR, Wang X, Guo C, Liu L, Cui R et al (2014). Bidirectional modulation of incubation of cocaine craving by silent synapse-based remodeling of prefrontal cortex to accumbens projections. Neuron 83: 1453-1467.

Mameli M, Halbout B, Creton C, Engblom D, Parkitna JR, Spanagel $\mathrm{R}$ et al (2009). Cocaine-evoked synaptic plasticity: persistence in the VTA triggers adaptations in the NAc. Nat Neurosci 12: 1036-1041.

McCutcheon JE, Wang X, Tseng KY, Wolf ME, Marinelli M (2011). Calcium-permeable AMPA receptors are present in nucleus accumbens synapses after prolonged withdrawal from cocaine self-administration but not experimenter-administered cocaine. J Neurosci 31: 5737-5743.

Meredith GE, Agolia R, Arts MPM, Groenewegen HJ, Zahm DS (1992). Morphological differences between projection neurons of the core and shell in the nucleus accumbens of the rat. Neuroscience 50: 149-162.

Neumann PA, Wang Y, Yan Y, Wang Y, Ishikawa M, Cui R et al (2016). Cocaine-induced synaptic alterations in thalamus to nucleus accumbens projection. Neuropsychopharmacology 41: 2399-2410.

Pacchioni AM, Gabriele A, See RE (2011). Dorsal striatum mediation of cocaine-seeking after withdrawal from short or long daily access cocaine self-administration in rats. Behav Brain Res 218: 296-300.

Parvaz MA, Moeller SJ, Goldstein RZ (2015). Incubation of cueinduced craving in human cocaine addiction measured by EEG. Poster, American College of Neuropsychopharmacology Annual Meeting.

Pascoli V, Terrier J, Espallergues J, Valjent E, O'Connor EC, Luscher C (2014). Contrasting forms of cocaine-evoked plasticity control components of relapse. Nature 509: 459-464.

Paxinos G, Watson C (2007). The Rat Brain in Stereotaxic Coordinates, 6th edn. Elsevier: Amsterdam, The Netherlands; Boston, MA.

Pickens CL, Airavaara M, Theberge F, Fanous S, Hope BT, Shaham Y (2011). Neurobiology of the incubation of drug craving. Trends Neurosci 34: 411-420.

Purgianto A, Scheyer AF, Loweth JA, Ford KA, Tseng KY, Wolf ME (2013). Different adaptations in AMPA receptor transmission in the nucleus accumbens after short vs long access cocaine selfadministration regimens. Neuropsychopharmacology $\mathbf{3 8}$ : 1789-1797.

Robinson TE, Gorny G, Mitton E, Kolb B (2001). Cocaine self-administration alters the morphology of dendrites and 
dendritic spines in the nucleus accumbens and neocortex. Synapse 39: $257-266$.

Robinson TE, Kolb B (1999). Alterations in the morphology of dendrites and dendritic spines in the nucleus accumbens and prefrontal cortex following repeated treatment with amphetamine or cocaine. Eur J Neurosci 11: 1598-1604.

Robinson TE, Kolb B (2004). Structural plasticity associated with exposure to drugs of abuse. Neuropharmacology 47(Suppl 1): 33-46.

Russo SJ, Dietz DM, Dumitriu D, Morrison JH, Malenka RC, Nestler EJ (2010). The addicted synapse: mechanisms of synaptic and structural plasticity in nucleus accumbens. Trends Neurosci 33: 267-276.

Sala C, Segal M (2014). Dendritic spines: the locus of structural and functional plasticity. Physiol Rev 94: 141-188.

Self DW, Choi KH, Simmons D, Walker JR, Smagula CS (2004). Extinction training regulates neuroadaptive responses to withdrawal from chronic cocaine self-administration. Learn Mem 11: 648-657.

Sesack SR, Grace AA (2010). Cortico-basal ganglia reward network: microcircuitry. Neuropsychopharmacology 35: 27-47.

Shen H-W, Gipson CD, Huits M, Kalivas PW (2014). Prelimbic cortex and ventral tegmental area modulate synaptic plasticity differentially in nucleus accumbens during cocaine-reinstated drug seeking. Neuropsychopharmacology 39: 1169-1177.
Soares C, Lee KF, Nassrallah W, Beique JC (2013). Differential subcellular targeting of glutamate receptor subtypes during homeostatic synaptic plasticity. J Neurosci 33: 13547-13559.

Stankeviciute NM, Scofield MD, Kalivas PW, Gipson CD (2014). Rapid, transient potentiation of dendritic spines in contextinduced relapse to cocaine seeking. Addict Biol 19: 972-974.

Terrier J, Luscher C, Pascoli V (2015). Cell-type specific insertion of GluA2-lacking AMPARs with cocaine exposure leading to sensitization, cue-induced seeking, and incubation of craving. Neuropsychopharmacology 41: 1779-1789.

Wang X, Cahill ME, Werner CT, Christoffel DJ, Golden SA, Xie Z et al (2013). Kalirin-7 mediates cocaine-induced AMPA receptor and spine plasticity, enabling incentive sensitization. J Neurosci 33: 11012-11022.

Wheeler RA, Carelli RM (2009). Dissecting motivational circuitry to understand substance abuse. Neuropharmacology 56(Suppl 1): 149-159.

Wolf ME (2016). Synaptic mechanisms underlying persistent cocaine craving. Nat Rev Neurosci 17: 351-365.

Wolf ME, Ferrario CR (2010). AMPA receptor plasticity in the nucleus accumbens after repeated exposure to cocaine. Neurosci Biobehav Rev 35: 185-211.

Wolf ME, Tseng KY (2012). Calcium-permeable AMPA receptors in the VTA and nucleus accumbens after cocaine exposure: when, how, and why? Front Mol Neurosci 5: 72.

Supplementary Information accompanies the paper on the Neuropsychopharmacology website (http://www.nature.com/npp) 